Conclusion We identified a subgroup of patients with hoarseness with objective detection of oesophago-pharyngeal reflux (10/21). (b) the majority of oropharyngeal $\mathrm{pH}$ drops detected by $\mathrm{Dx}-\mathrm{pH}$ do not correlate with retrograde flow (liquid or gas) in the oesophageal body. (c) Detection of pepsin in saliva suggests the likelihood of reflux episodes in the previous $60 \mathrm{~min}$.

Competing interests None declared.

\section{PTU-186 EXOGENOUS NSAID EXPOSURE INCREASES TRANSCRIPTIONAL EXPRESSION OF THE 5-LIPOXYGENASE PATHWAY IN NORMAL SQUAMOUS TISSUE, BUT NOT IN BARRETT'S METAPLASIA: AN EX VIVO STUDY OF OESOPHAGEAL BIOPSY SPECIMENS}

doi:10.1136/gutjnl-2012-302514c.186

\begin{abstract}
1,2 J Neale, ${ }^{*}{ }^{1,2} \mathrm{C}$ P C Boger, ${ }^{1,2} \mathrm{~J}$ Shutt, ${ }^{1} \mathrm{P}$ Patel, ${ }^{3} \mathrm{~J}$ W Holloway, ${ }^{2} \mathrm{~A}$ P Sampson. ${ }^{1}$ Gastroenterology, Southampton Hospitals NHS Trust, Southampton, UK; ${ }^{2}$ Clinical and Experimental Sciences Academic Unit, University of Southampton School of Medicine, Southampton, UK; ${ }^{3}$ Human Development \& Health Academic Unit, University of Southampton School of Medicine, Southampton, UK
\end{abstract}

Introduction Barrett's oesophagus (BO) is a premalignant condition for oesophageal adenocarcinoma, an increasingly common malignancy with poor survival. The prostanoids and leukotrienes are lipid inflammatory mediators derived from a common precursor by the cyclooxygenase (COX) and 5-lipoxygenase (5-LO) pathways respectively. Both families have been implicated in oesophageal adenocarcinoma, prompting studies of the effects on disease progression of drugs that modulate these pathways. NSAIDs may prevent tumorigenesis by inhibiting COX, but are also a risk factor for reflux and Barrett's disease. Increased expression of 5-LO pathway proteins is implicated in oesophageal adenocarcinoma, so we hypothesised that in vitro exposure of oesophageal tissue to an NSAID may induce transcriptional expression of genes for 5-LO pathway enzymes.

Methods Patients with known BO $(n=14)$ were recruited as they attended endoscopy for routine surveillance. There was no evidence of dysplasia before or after this endoscopy. In each patient, oesophageal biopsies were taken from a single level of $\mathrm{BO}$ and from proximal squamous mucosa. Biopsies from each site were cultured for $15 \mathrm{~h}$ at $37^{\circ} \mathrm{C}$ in HEPES-buffered DMEM in the presence or absence of indomethacin $(10 \mu \mathrm{M})$. Biopsies were then transferred to lysis buffer for quantification of mRNA for 5-LO, FLAP, $\mathrm{LTA}_{4}$ hydrolase and $\mathrm{LTC}_{4}$ synthase using quantitative RT-PCR. Transcript levels for each enzyme were calculated as mean $\Delta \Delta \mathrm{Ct}$ values compared to baseline expression after correction with a calibration sample across all plates.

Results Culture with indomethacin $(10 \mu \mathrm{M}, 15 \mathrm{~h})$ significantly increased transcriptional expression of 5-LO $(p<0.01)$ and FLAP $(p<0.05)$ in squamous control biopsies, with a trend for increased LTC $_{4}$ synthase transcripts $(p=0.08)$ but not LTA $_{4}$ hydrolase $(p=0.38)$, suggesting induction of a cysteinyl-leukotriene biosynthetic pathway. Expression of the 5-LO pathway genes was elevated at baseline in Barrett's tissues and in vitro exposure to indomethacin did not produce further changes in this group (all $\mathrm{p}>0.2$ ).

Conclusion These results indicate that indomethacin increases transcriptional expression of the proximal, rate-limiting enzymes of the 5-LO pathway in squamous oesophageal tissues, which may lead to increased enzyme activity and production of tumorigenic leukotrienes. This preliminary study suggests that brief NSAID exposure in vitro may mimic changes in the expression of 5-LO pathway genes induced in vivo in Barrett's metaplasia.

Competing interests None declared.
PTU-187 LONG-TERM QUALITY OF LIFE AFTER OESOPHAGECTOMY FOR CANCER: COMPARISON OF CERVICAL VS MEDIASTINAL ANASTOMOSES

doi:10.1136/gutjnl-2012-302514c.187

1J M H Bennett, ${ }^{2}$ J Wormald, ${ }^{3} \mathrm{M}$ Van Leuvan, ${ }^{1} \mathrm{M}$ Lewis. ${ }^{1}$ General and Upper G/ Surgery, Norfolk and Norwich University Hospital, Norwich, UK; ${ }^{2}$ UEA Medical School, UEA, Norwich, UK; ${ }^{3}$ Thoracic Surgery, Norfolk and Norwich University Hospital, Norwich, UK

Introduction With improvements in neoadjuvant therapy and earlier diagnosis, long-term survival after oesophagectomy for adenocarcinoma is becoming more frequent. With longer survival the quality of life (QOL) of patients post resection has become a greater priority. There has been extensive debate focusing on the long term effects of different sites for anastomosis. We aimed to examine if post-oesophagectomy QOL is affected by the site of the surgical anastomosis. Methods OORTC C-30 and OG-25 QOL questionnaires were analysed from post-oesophagectomy patients surviving $>3$ years. Data were available both from a prospective database and questionnaires sent to post-oesophagectomy patients. Surgery was completed by two surgical teams and data were analysed in subsets dependant on the site of oesophago-gastric anastomosis-either thoracic or cervical. No patients underwent formal pyloroplasty. Data were analysed using the Student t test on SPSS statistical software. QORTC C-30 data were compared against the reference tables for oesophageal cancer pre-treatment and a cohort of presurgical patients awaiting oesophagectomy. Ethical approval was granted by the local MREC.

Results A total of 60 patients responded ( $82 \%$, anastomosis: thoracic $\mathrm{n}=29$, cervical $\mathrm{n}=31$ ) with a median time post-surgery of 6.1 years (range 3-12 years). Cervical and thoracic anastomosis subgroups were equivalent in terms of age at time of surgery, time post op and cancer stage. No significant QOL difference was noted between cervical or chest anastomosis groups for any functional or symptom score, especially focusing on dysphagia (OG25, $\mathrm{p}=0.24$ ), odynophagia (OG 25, $\mathrm{p}=0.68$ ) and swallowing problems (OG25, $\mathrm{p}=0.73$ ). The patients' overall general health (OL2) score was $72.0 \pm 19.43$ (mean \pm SD) compared with $74.8 \pm 20.57$ for a cohort of $53 \mathrm{NNUH}$ pre-op oesophagectomy patients and $71.2 \pm 22.4$ for the QORTC general population reference data. Functional indices and symptom scores are improved for our cohort compared to the QORTC oesophageal cancer reference baseline except symptom scores for diarrhoea and dyspnoea which worsen post-operatively.

Conclusion There is no significant difference in QOL scores between oesophagectomy patients with cervical or thoracic anastomosis at $>3$ years post-surgery when analysed using the QORTC C-30 and OG25 questionnaires. QOL in long-term survivors after oesophagectomy compares favourably with QORTC reference data for both pre-treatment oesophageal cancer and baseline general population data in our cohort, possibly due to the absence of pylorplasty. Further prospective QOL data collection is required to elucidate any long-term differences between the two anastomosis sites.

Competing interests None declared.

\section{PTU-188 "KNITTED" OESOPHAGEAL STENTS: SUPERIOR CONFORMABILITY WITH NO TRADE OFF}

doi:10.1136/gutjnl-2012-302514c.188

1J R Cain, ${ }^{*} \mathrm{~T}$ Westwood, ${ }^{2} \mathrm{C}$ Gordon, ${ }^{3} \mathrm{R}$ A Frost, ${ }^{1} \mathrm{H}-\mathrm{U}$ Laasch. ${ }^{1}$ Radiology, The Christie NHS Foundation Trust, Manchester, UK; ${ }^{2}$ Gastroenterology, The Royal Bournemouth and Christchurch Hospitals NHS Foundation Trust, Bournemouth, UK; ${ }^{3}$ Radiology, Salisbury NHS Foundation Trust, Salisbury, UK

Introduction Knitted enteral stents have reduced stent shortening and no axial straightening forces, resulting in better conformability 
to tortuous anatomy compared to conventional braided stents. The first covered removable oesophageal stent is now available consisting of a knitted Nitinol skeleton with large heads covered in silicone, but the shaft only externally covered by an ePTFE membrane. In vitro it has better conformability but comparable radial force to standard braided stents. We report the first experience world-wide with this type of device.

Methods All consecutive patients over a 9-month period referred for oesophageal stenting had an Egis stent (BVM Medical, Hinckley, UK/S\&G Biotech, Seoul, Korea) placed under fluoroscopic guidance. Stents with anti-reflux valve were used if the lower end had to be placed in the stomach. Patients were followed up prospectively until death or stent failure. Clinical outcome was compared to the results from the national Registry of Oesophageal Stenting (ROST). The device is CE marked and was used within its license. Institutional review board was nevertheless sought and granted. The manufacturer made 30 stents were available for evaluation free of charge. Results 22 patients with malignant dysphagia and 2 with a benign post-radiation stricture had an EGIS oesophageal stent inserted. Median immediate stent expansion at insertion was 45\% (25\%$100 \%)$ increasing to $100 \%(60 \%-100 \%)$ after 1 week. Minor complications occurred in $8 \%$ compared to $14 \%$ in the national audit. No stent migrated from the oesophagus above the cardia, partial migration occurred in $1 / 15$ stents (9.8\%) placed across the GO-junction, comparing favourably to a migration rate of $4.3 \%$ (mid-oesophagus) and $18 \%$ (cardia) from the national audit. Improvement in dysphagia was comparable with a pre-stent median score of $3(2-4)$ improving to $2(0-3)$ at $48 \mathrm{~h}$ and to $1(1-3)$ after 1 week. The two stents inserted for benign strictures were removed endoscopically by inversion through capture of the lower purse string. Initial difficulties with the delivery system were identified and corrected by the manufacturer.

Conclusion Limited first experience shows the EGIS oesophageal stent to perform at least as good as the large variety of existing oesophageal stents. There may be a benefit in terms of reduced stent migration, particularly if placed across the adverse anatomy of the GO-junction, but more extensive experience is required. Palliation of dysphagia is as good as with conventional stents. The stent should be particularly considered in tortuous anatomy due to the excellent conformability.

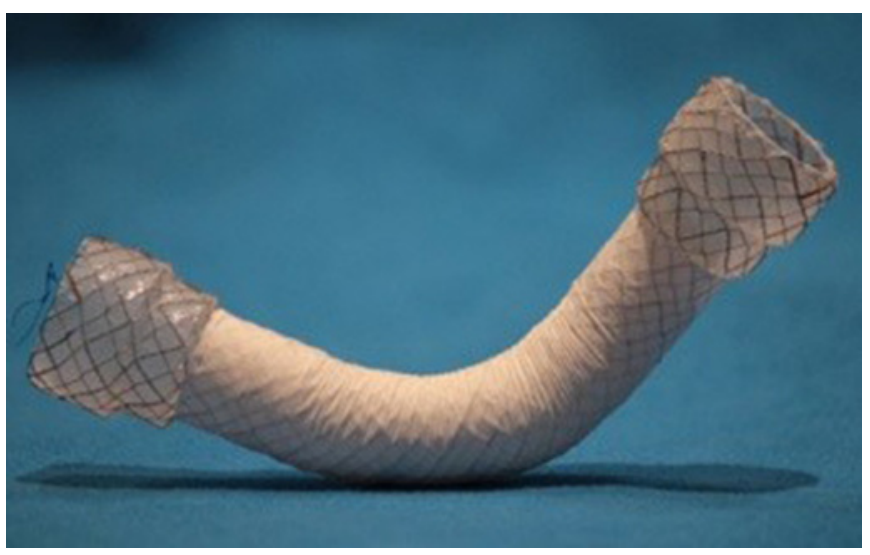

Abstract PTU-188 Figure 1

Competing interests J Cain: None declared, T Westwood: None declared, C Gordon: None declared, R Frost: None declared, H-U Laasch Consultant for: S\&G Biotech.

\section{REFERENCE}

1. British Society of Interventional Radiology (BSIR). ROST - Registry of Oesophageal Stenting, First Report 2004. Henley-on-Thames: Dendrite Clinical Systems, 2004

\section{PTU-189 ARE THE CURRENT MANAGEMENT STRATEGIES FOR T1/T2 NO OESOPHAGEAL CANCER OPTIMAL?}

doi:10.1136/gutjnl-2012-302514c.189

J Evans, ${ }^{*}$ R Pande, D Beardsmore, D Corless, W Crisp, C Cheruvu. University Hospital North Staffordshire, Stoke-On-Trent, UK

Introduction Currently patients with Stage 1 (T1/T2 N0) oesophageal cancer proceed straight to surgery; these patients have an expected $60 \%-90 \% 5$ year survival. This staging is based on EUS (endoscopic ultrasound) and CT imaging, which are accurate in $90 \%$ and $70 \%$ for $\mathrm{T}$ stage respectively and $70 \%$ in $\mathrm{N}$ stage for EUS. However, reporting of postoperative histology reveals a significant proportion of these patients end up with locally advanced (T3 N1/0) disease, leading to a 5 -year survival of $25 \%$. The aim of this study is to assess the results based on the current UK protocols.

Methods This study includes all patients who underwent an oesophagectomy over a 4-year period (2008-2011), focusing on those with T1/T2 N0 disease. After comprehensive staging which included endoscopy, EUS, CT and PET scanning all patients results were reviewed. Data on patients with T1/T2 N0 disease who went straight to surgery were analysed. The primary end points included a review of the number of patients who had a higher staging on post-operative histology and also the peri-operative mortality, morbidity and survival.

Results 167 oesophagectomies were carried out during the study period in our institution, of which $26(15.6 \%)$ were for preoperatively staged T1/T2 N0 disease (6 T1 and $20 \mathrm{~T} 2)$. The mean age was 68 years and $18(69.2 \%)$ of the patients were male. Ivor-Lewis oesophagectomies were performed on 23 patients, minimally invasive 3 -stage procedures in 2 and 1 had a trans-hiatal operation. Postoperative histology indicated a higher stage of disease in 54\% (14) of patients, 10 had a T3 lesion while 10 patients were noted to have nodal disease. One (3.8\%) patient died on the 8th post-op day with bowel ischaemia and major or minor complications occurred in 11 (42\%) of patients peri-operatively. Disease recurrence was seen in 4 $(15.3 \%)$ patients at 15 to 18 months post-operatively, $3(75 \%)$ of these had stage migration on histology to a higher stage and two have died.

Conclusion Our study concludes that in patients with T1/2 N0 oesophageal cancer, despite the high specificity and sensitivity of both EUS and CT scan we still seem to have a large proportion of patients who are under staged and often offered curative operations, which appear to result in sub-optimal management leading to early recurrence and poorer long-term outcomes. Further studies are essential for accurate assessment of this stage of disease so that appropriate treatment strategies can be implemented for the management of oesophageal cancer.

Competing interests None declared.

\section{PTU-190 ISOLATED UPRIGHT OESOPHAGEAL ACID EXPOSURE IS ASSOCIATED WITH LESS FAVOURABLE OUTCOMES AFTER LAPAROSCOPIC ANTI-REFLUX SURGERY}

doi:10.1136/gutjnl-2012-302514c.190

J A Cornish, ${ }^{*}$ E O'Dea, C Thomas, G W Clark. General Surgery, University Hospital of Wales, Cardiff, UK

Introduction With the increasing use of antireflux surgery since the widespread dissemination of the laparoscopic approach, preoperative indicators to predict success could reduce the number of patients with a poor outcome. The aim of this study was to identify if poorer outcomes could be predicted in patients with upright reflux alone vs mixed upright and supine reflux, in the presence of a positive DeMeester score. 